\title{
Wirtschaftliche Auswirkungen der direkten Demokratie
}

\author{
Gebhard Kirchgässner* \\ Universität St. Gallen
}

\section{Einleitung}

Die direkte Demokratie scheint derzeit im Aufwind zu sein. Dies gilt zunächst für die Schweiz und für die Vereinigten Staaten. So hat in der Schweiz, dem Staat mit den am stärksten ausgebauten Volksrechten, die Zahl der auf nationaler Ebene durchgeführten Volksabstimmungen in den letzten Jahrzehnten erheblich zugenommen. Waren es in den dreißiger Jahren insgesamt 21 Abstimmungen über Referenden und Initiativen, so wurden die Stimmbürgerinnen und Stimmbürger in den neunziger Jahren insgesamt neunzigmal zur Urne gerufen. ${ }^{1}$ Auch in den Vereinigten Staaten, die zwar nicht auf nationaler Ebene, wohl aber in einer Reihe von Bundesstaaten, direkte Volksrechte kennen, erfreuen sich Referenden und Initiativen zunehmender Beliebtheit. So wurde im Jahr 1993 die Volksinitiative neu in die Verfassung der Staates Mississippi aufgenommen. In den Staaten New Jersey, Rhode Island und Texas wird die Einführung der Volksinitiative ebenfalls erwogen. Daneben werden - wie in der Schweiz - die bestehenden Volksrechte zunehmend stärker genutzt. So wurde in Kalifornien allein von 1990 bis einschließlich 1996 über insgesamt 49 Initiativen abgestimmt, in den gesamten sechziger Jahren jedoch nur über 9 Initiativen. $^{2}$

Auch in der Bundesrepublik Deutschland ist die direkte Demokratie im Aufwind. Selbst auf Bundesebene ist die Forderung nach der Einführung direktdemokratischer Elemente heute nicht mehr alleine eine Angelegenheit (außerparlamentarischer) politischer Außenseiter. Sie wurde vielmehr sogar in der nach der deutschen Vereinigung eingesetzten 'Gemeinsamen Verfassungskommission' erörtert. Vorgesehen war ein abgestuftes Verfahren von Volksinitiative, Volksbegehren und Volksentscheid. ${ }^{3}$ Mit der Volksinitiative sollten die Bürgerinnen und Bürger die Möglichkeit erhalten, den Deutschen Bundestag zur Behandlung bestimmter Gegenstände und Gesetzesentwürfe zu zwingen.

\footnotetext{
* Für Anregungen und Hinweise danke ich Lars P. Feld (Universität St. Gallen).

1. Siehe: Statistisches Jahrbuch der Schweiz 2000, Tabelle 17.13, S. 454.

2. Siehe hierzu Heussner (1999, S. 7).

3. Siehe hierzu Luthardt und Waschkuhn (1997, S. 63ff.).
}

(C) Verein für Socialpolitik und Blackwell Publishers Ltd. 2000, 108 Cowley Road, Oxford OX4 1JF, UK und 350 Main Street, Malden, MA 02148, USA. 


\section{Gebhard Kirchgässner}

Falls er einem solchen Entwurf nicht innerhalb einer bestimmten Frist zugestimmt hätte, hätte die Möglichkeit bestanden, ein Volksbegehren einzuleiten. Wäre hierfür die erforderliche Unterschriftenzahl erreicht worden, so hätte ein Volksentscheid stattgefunden, und bei Erreichung der notwendigen (möglicherweise qualifizierten) Mehrheit wäre der Gesetzentwurf angenommen gewesen.

Die entsprechenden, von der SPD und von der Partei „Bündnis 90/Die Grünen" stammenden Anträge hatten in der Kommission bei der Abstimmungssitzung am 11. Februar 1993 zwar eine knappe Mehrheit erhalten, aber nicht die für eine Empfehlung notwendige Zweidrittelmehrheit. Für die Volksinitiative hatten sich bei einer vorangehenden Anhörung andererseits sieben von neun Sachverständigen ausgesprochen, so dass die generelle Ablehnung der Aufnahme direkt demokratischer Instrumente in das Grundgesetz durch diese Kommission „doch etwas überraschend kam“, wie Luthardt und Waschkuhn (1997, S. 64) zu Recht schreiben. ${ }^{4}$ Wenn es heute möglich wäre, in der Bundesrepublik Deutschland eine Abstimmung über die Einführung der direkten Demokratie auf Bundesebene durchzuführen, könnte man mit einer Mehrheit der Bevölkerung rechnen. Zudem enthalten heute alle Länderverfassungen direkte Volksrechte. Dies gilt für die alten wie für die neuen Bundesländer. ${ }^{5}$ Da die Quoren in den meisten Landesverfassungen (immer noch) recht hoch angesetzt sind, konnte in der Bundesrepublik Deutschland bisher jedoch noch nicht viel Erfahrung mit der direkten Demokratie gesammelt werden.

In der Schweiz gibt es andererseits seit vielen Jahren eine eher kritische Einschätzung der Auswirkungen der direkten Demokratie, welche vor allem von Ökonomen vorgebracht wird. ${ }^{6}$ Sie gilt als langsam und ineffizient. Dies wird insbesondere in der heutigen Zeit als ein Problem angesehen, da von der Politik rasche Entscheidungen verlangt würden. Die zur Vermeidung von Niederlagen in Referenden notwendige Suche nach Kompromissen, die möglichst viele Betroffene einschließen, mache zudem eine klare wirtschaftspolitische Strategie unmöglich. ${ }^{7}$

Tatsächlich lässt sich zeigen, dass direkte Volksrechte erheblichen Einfluss auf den politischen Prozess haben. Dies wird im übrigen auch kaum bestritten. Umstritten ist jedoch, ob dieser Einfluss positiv oder negativ zu bewerten ist. So heben die Gegner der direkten Demokratie neben der Langsamkeit der

4. Zur Diskussion in der Gemeinsamen Verfassungskommission siehe auch Schmack-Reschke (1997).

5. Zur Übersicht siehe Luthardt und Waschkuhn (1997) sowie Jung (1998); eine ausführliche Übersicht über den Stand Mitte 1992 sowie über die Praxis bis zu diesem Zeitpunkt gibt Jürgens (1993).

6. Siehe z.B. Borner, Brunetti und Straubhaar (1990, 1994), Kleinewefers (1997) oder Wittmann (1998).

7. Wie zuletzt die Ausführungen von Lucas Mühlemann, dem Vorsitzenden des Verwaltungsrats der Credit Suisse, im Tagesanzeiger-Magazin vom 31. Dezember 1999 zeigten, finden solche Überlegungen in den Chefetagen der schweizerischen Wirtschaft erheblichen Widerhall. 


\section{Wirtschaftliche Auswirkungen der direkten Demokratie}

Entscheidungsprozesse insbesondere den Einfluss hervor, den (kleine) Interessengruppen mit Hilfe des Referendums erringen können. Zudem seien die Bürgerinnen und Bürger in den meisten Fällen gar nicht in der Lage, politische Entscheidungen sachkundig $\mathrm{zu}$ treffen. Dagegen führen die Befürworter die höhere Qualität des politischen Diskurses sowie positive wirtschaftliche Auswirkungen ins Feld. ${ }^{8}$

Im folgenden soll zunächst die Frage aufgenommen werden, inwieweit die Stimmbürger unter verschiedenen institutionellen Bedingungen in der Lage sind, politische Entscheidungen sachkundig zu treffen, d.h. es geht um die Informationsvermittlung in der direkten wie der repräsentativen Demokratie (Abschnitt 2). Dabei wird sich zeigen, dass die Bürgerinnen und Bürger in der direkten Demokratie im allgemeinen besser informiert sind als im rein repräsentativen System, weil sowohl die Nachfrage als auch das Angebot an Information größer sind. In Abschnitt 3 wird ein kurzer Überblick über empirische Studien gegeben, welche wirtschaftliche Auswirkungen der direkten Demokratie untersuchen. Diese Arbeiten zeigen, dass die wirtschaftlichen Auswirkungen eher positiv sind. Abschließend wird noch auf einige Argumente eingegangen, die in Deutschland häufig gegen die direkten Volksrechte vorgebracht werden, insbesondere auf den Verweis auf die (angeblich) schlechten Erfahrungen, die in der Weimarer Republik damit gemacht wurden (Abschnitt 4).

\section{Informationsvermittlung in der repräsentativen und der direkten Demokratie}

Es ist eine alte Erkenntnis der Politikwissenschaft, auf die schon Jean-Jacques Rousseau und Thomas Jefferson aufmerksam gemacht haben, dass ein hoher Informationsstand der Bürgerinnen und Bürger eine Voraussetzung für die Funktionsfähigkeit einer Demokratie ist. Damit sie nämlich die „richtigen“ Entscheidungen treffen können, d.h. jene Entscheidungen, welche ihren Präferenzen am ehesten entsprechen, müssen sie über die zur Entscheidung stehenden Alternativen gut informiert sein. Hierzu ist nicht nur die Kenntnis ihrer eigenen Präferenzen, sondern vor allem „Sachkenntnis“ erforderlich. Dies gilt in der repräsentativen Demokratie für die Wahl eines Abgeordneten oder einer Partei genauso wie in der direkten Demokratie für die Annahme oder Ablehnung einer bestimmten Vorlage.

Dem steht entgegen, dass die Wählerinnen und Wähler in einer Demokratie kaum Anreize haben, sich zu informieren. Sobald sich bei einer Wahl oder Abstimmung nur ganz wenige Bürger beteiligen, ist die Wahrscheinlichkeit, dass eine einzelne Stimme den Ausschlag gibt, praktisch Null. Damit aber hat der/die einzelne Stimmbürger(in) in großen Gemeinden, auf Landes- (Kantons-)

8. Siehe z.B. Frey und Kirchgässner (1993), Feld und Savioz (1997), Feld und Kirchgässner (1999) oder Kirchgässner, Feld und Savioz (1999). 


\section{Gebhard Kirchgässner}

und erst recht auf Bundesebene mit seiner/ihrer Stimme keinerlei Einfluss auf das Gesamtergebnis. Daher stellt sich zunächst die Frage, weshalb sich ein rationales Individuum überhaupt an einer Wahl oder Abstimmung beteiligen sollte. ${ }^{9}$ Aber selbst wenn jemand, aus welchen Gründen auch immer, sich beteiligt, gibt es für den einzelnen keinen Grund, sich besonders zu informieren. Die Beschaffung und Verarbeitung von Information ist mit Kosten verbunden. Solche Kosten nehmen rationale Individuen nur dann auf sich, wenn sie sich davon einen entsprechenden Nutzen versprechen. Solange das eigene Handeln aber keinen Einfluss auf das Gesamtergebnis hat, ist ein solcher Nutzen nicht erkennbar. Wie bereits Downs (1957, S. 233ff.) festgestellt hat, ist der rationale Bürger daher ein „rationaler Ignorant“.

Wir befinden uns damit in einem Dilemma: Demokratie setzt für ihre Funktionsfähigkeit den informierten Bürger voraus, aber sie vermittelt keine (oder zumindest zu wenige) Anreize dafür, dass sich die Bürgerinnen und Bürger informieren. Soweit eine Lösung überhaupt gefunden werden kann, kann sie nur darin liegen, dass die Informationskosten für die Bürger soweit als möglich gesenkt werden. Nach Downs (1957, S. 93ff.) ist dies eine wesentliche Funktion, welche Ideologien in der repräsentativen Demokratie haben. Auch mit Hilfe der modernen elektronischen Medien ist es unmöglich, im Rahmen einer Wahlkampagne den Bürgern alle wesentlichen Informationen über die von einer Regierung oder Partei geplanten Vorhaben zu vermitteln. Man versucht daher, die „ideologische“ Position einer Partei oder eines Kandidaten den Wählern nahezubringen. Diese sollen wissen, „wofür ein(e) Kandidat(in) oder eine Partei steht", und sie sollen sich darin wiederfinden können, ohne dass sie über alle Einzelheiten der geplanten Politik informiert sein müssen.

Selbstverständlich wird auch Sachinformation vermittelt. Schließlich benötigt man zur Vermittlung eines ideologischen Profils zumindest ein Minimum an Sachinformation. Aber auch diese Information wird ihre Abnehmer um so eher erreichen, je „kostengünstiger" sie angeboten wird. Kostengünstig kann Information durch die Medien, insbesondere durch das Fernsehen angeboten werden. Wer am Abend die Nachrichtensendung sieht, nimmt eine Menge politischer Informationen auf, ohne dass ihr/ihm dabei irgendwelche Kosten entstehen. Schaffen es ein Politiker oder eine Partei, in diesen Nachrichten positiv erwähnt zu werden, so wird eine für ihn/sie positive Information den Bürgern vermittelt. Kann solche Information überdies im Rahmen einer Veranstaltung vermittelt werden, die für sich genommen bereits Unterhaltungswert besitzt, so wird es um so leichter sein, Sachinformationen zu vermitteln. Die Verbreitung von Information ist dabei allerdings nur Nebenprodukt. Dies gilt insbesondere für die großen Fernsehdebatten, wie wir sie vor allem vor Wahlen kennen. Zumindest bei den kommerziellen (amerikanischen) Fernsehanstalten ist es dabei oberstes Interesse, hohe Einschaltquoten zu erzielen, um die Werbeeinnahmen zu steigern. Hauptinteresse der Kandidaten ist die Vermittlung ihrer ideologischen Position, um die

9. Siehe hierzu z.B. Kirchgässner (1990). 


\section{Wirtschaftliche Auswirkungen der direkten Demokratie}

entsprechenden Wählerschichten an sich zu binden. Das Publikum schließlich erwartet vor allem eine interessante Show, d.h. Unterhaltung. Alle drei Ziele können im allgemeinen jedoch nur dann erreicht werden, wenn nicht nur über persönliche Charakteristika der Kandidaten, sondern auch über Sachfragen diskutiert wird.

Aber auch ohne solche direkte Vermittlung steht den Wählern Information zur Verfügung. Es hat sich gezeigt, dass die Wähler bei ihrer Entscheidung vor allem rückwärts blicken und die bisherige Leistung einer Regierung beurteilen („retrospective voting“) und dass sie weniger nach vorne blicken und Parteiund Regierungsprogramme miteinander vergleichen. Wenn sie die Leistung einer Regierung am Ende einer Legislaturperiode beurteilen, schätzen sie ab, in welchem Maße sie durch die Handlungen dieser Regierung positiv oder negativ betroffen wurden. Sind sie mit der Leistung der Regierung zufrieden, werden sie eher für die Regierung (bzw. für eine der Regierungsparteien) stimmen, sind sie dagegen unzufrieden, werden sie eher für die Opposition (bzw. für eine der Oppositionsparteien) stimmen. Voraussetzung dafür, dass Information hier wirksam werden kann, ist, dass zum einen ein entsprechend großer Teil der Bevölkerung mit solcher Information persönlich konfrontiert wird und dass die Wähler zweitens die Regierung für die entsprechende Entwicklung verantwortlich machen. ${ }^{10}$

Dies kann am Beispiel der Wirtschaftspolitik verdeutlicht werden. Es gibt eine Reihe von Studien, die zeigen, dass die Entscheidung der Wähler für oder gegen eine Partei bzw. Regierung sowie die mit Hilfe von Meinungsumfragen zwischen Wahlterminen gemessene Wahlabsicht der Bevölkerung wesentlich von der Entwicklung der wirtschaftlichen Lage abhängt. ${ }^{11}$ Untersucht man, welche Variablen hier eine Rolle spielen, so sind dies vor allem die Arbeitslosenquote, die Inflationsrate und die Zuwachsrate des verfügbaren (realen) Einkommens, d.h. es sind Variablen, über deren Entwicklung die Individuen in ihrem täglichen Leben (als Konsumenten und Arbeitnehmer) Information erhalten und die sie auch direkt betreffen. Andere, ebenfalls 'wichtige' wirtschaftliche Variablen, über die sie nur über die Medien informiert werden und die sie außerdem persönlich nur indirekt betreffen, wie z.B. die Situation der Zahlungsbilanz oder die Höhe und Entwicklung der Staatsverschuldung, scheinen dagegen keinen Einfluss zu haben.

Dadurch, dass zum einen für Wahlen weniger Information notwendig scheint als für Sachabstimmungen und dass zweitens Vertreter (Repräsentanten) bei schwierigen Sachfragen besser informiert entscheiden können, erhält die repräsentative Demokratie gegenüber der direkten Demokratie eine informationstheoretische Rechtfertigung: Es wird unterstellt, dass die Bürger bei direkten Sachentscheidungen wegen der Komplexität dieser Entscheidungen häufig oder sogar fast immer überfordert sind.

10. Siehe hierzu Kirchgässner (1986, S. 426).

11. Für eine neuere Übersicht über diese Literatur siehe Nannestad und Paldam (1994), speziell für die Bundesrepublik Deutschland Kirchgässner (1986). 


\section{Gebhard Kirchgässner}

Tatsächlich benötigen die Bürgerinnen und Bürger in der direkten Demokratie deutlich mehr Information als in der repräsentativen Demokratie. Schließlich sollen sie über einzelne Gesetzesvorlagen direkt entscheiden. Bei der Annahme oder Ablehnung von Vorlagen wird gelegentlich die Auffassung vertreten, mangelnde Information über die sich aus einer Ablehnung bzw. Annahme ergebenden Konsequenzen habe $\mathrm{zu}$ einer (aus der Sicht des Betrachters) 'falschen' Entscheidung geführt. Ganz allgemein aber stellt sich auch hier die Frage, ob und wie Anreize gesetzt werden können, damit die Bürger die notwendige Information erhalten, um im Sinne ihrer individuellen Präferenzen sachgerecht entscheiden zu können.

Im Vergleich mit der repräsentativen Demokratie spielen hier die Parteien eine untergeordnete Rolle. Wichtiger für den Entscheidungsprozess der Bürger sind die Stellungnahmen derjenigen, die durch die zur Diskussion stehenden Regelungen direkt betroffen werden, insbesondere der Interessengruppen. So hat Schneider (1985) gezeigt, dass die „Parolen“, d.h. die öffentlich abgegebenen Abstimmungsempfehlungen der Interessenverbände in der Schweiz, einen deutlich stärkeren Einfluss auf den Ausgang von Referenden haben als die Parolen der Parteien. ${ }^{12}$

Der im Vorfeld einer Volksabstimmung in der Schweiz stattfindende Diskurs ist von vier Eigenschaften geprägt: ${ }^{13}$

(i) Die Vorlagen werden immer im Vergleich zu Alternativen - meist dem Status quo - erörtert. Die Kosten der Durchführung sind ein wichtiger Bestandteil der Diskussion. Beides bewirkt, dass der Austausch der Argumente nicht auf allgemeiner und unverbindlicher Ebene bleibt.

(ii) Der Diskurs ist um so intensiver, je wichtiger eine Vorlage ist, d.h. je stärker sich die Bürger in den betreffenden Gebietskörperschaften, einer Gemeinde, einem Kanton oder dem Bund, davon betroffen fühlen. Die Inhalte mancher Volksabstimmungen werden als unbedeutend angesehen, so dass auch die Diskussion wenig intensiv ist. Bei anderen Abstimmungen erfolgt ein längerer und grundsätzlicherer Austausch von Argumenten. Der einem Urnengang vorangehende Diskurs ist somit nicht mechanisch festgelegt, sondern er ändert sich je nach der Bedeutung des Themas für die Stimmbürger.

(iii) Am Diskurs beteiligen sich sowohl Organisationen wie Parteien und Verbände als auch Einzelpersonen, und er findet auf den verschiedensten Niveaus der Gesprächskultur statt. Er ist nicht auf Intellektuelle beschränkt, sondern auch im Rahmen eines Sportklubs oder Stammtisches möglich. Auch die Beteiligung der Stimmbürgerinnen und Stimmbürger weist je

12. Zu einem ähnlichen Resultat gelangen Lupia (1994), sowie Bowler und Donovan (1998), für die USA. Sie stellen zudem fest, dass die Stimmbürger eher für eine Initiative stimmen, wenn diese von typischen Bürgerbewegungen unterstützt wird als von zahlungskräftigen Interessengruppen, wie etwa der amerikanischen Tabakindustrie.

13. Siehe hierzu auch Frey und Kirchgässner (1993). 


\section{Wirtschaftliche Auswirkungen der direkten Demokratie}

nach perzipierter subjektiver Bedeutung bzw. Betroffenheit erhebliche Schwankungen auf. So lag auf Bundesebene die durchschnittliche Beteiligung in den siebziger und achtziger Jahren bei 41,5 Prozent der Stimmberechtigten. ${ }^{14}$ Wie die Beispiele der Abstimmungen über den UNOBeitritt, die Abschaffung der Armee, den EWR-Beitritt und die Erhöhung des Treibstoffzolls zeigen, kann bei Entscheidungen, die als wichtig eingestuft werden, die Beteiligung jedoch deutlich darüber liegen. ${ }^{15}$

(iv) Im Verlauf dieses Diskurses findet zumindest bei Teilen der Bevölkerung ein Lernprozess statt. ${ }^{16}$ Dadurch, dass sie mit den Argumenten beider Seiten konfrontiert werden, sehen sich viele dazu veranlasst, die jeweilige Frage genauer zu überdenken. Dies kann dazu führen, dass einige und gelegentlich sogar viele ihre Auffassung ändern. Damit ergibt sich auch die Chance, dass die Stimmbürgerinnen und Stimmbürger ihre aktuell wirksamen Präferenzen in Richtung auf Verallgemeinbarkeit hinterfragen.

Man kann davon ausgehen, dass wegen der direkten Betroffenheit der Bürger die Bereitschaft, Informationskosten auf sich zu nehmen, in der direkten Demokratie größer ist als in der repräsentativen Demokratie. Dazu kommt, dass es für die Bürger auch privat wichtig werden kann, über politische Fragen, die zur Entscheidung anstehen, gut informiert zu sein. Dies geschieht dann, wenn andere Bürgerinnen und Bürger, mit denen man in Kontakt ist, erwarten, dass man gut informiert ist, und wenn die Enttäuschung dieser Erwartung zu einem Verlust an Prestige führen kann.

Aber auch hier gilt, dass die Informationen so aufbereitet werden müssen, dass sie von den Bürgern ohne allzu große Kosten aufgenommen und verarbeitet werden können. Und auch hier werden - wie bei Wahlen gelegentlich die „falschen“ Argumente obsiegen. Wichtig ist jedoch, dass sich die Argumente im politischen Diskurs auf Sachfragen beziehen und dass es kaum möglich ist, sich mit dem Herausarbeiten einer ideologischen Position zu begnügen. ${ }^{17}$ Diese reicht in aller Regel nicht aus, um die Bürger zu einer bestimmten Entscheidung zu motivieren. Dies wird insbesondere dann deutlich, wenn Anhänger einer Partei in größerer Zahl gegen die von ihrer Partei vertretene Linie stimmen, was nicht selten geschieht.

14. Siehe Statistisches Jahrbuch der Schweiz 1993, S. 376.

15. Die entsprechenden Werte der Stimmbeteilung sind: UNO-Beitritt (16.3.1986): 50,7 Prozent, die Abschaffung der Armee (26.11.1989): 62,9 Prozent, EWR-Beitritt (6.12.1992): 78,3 Prozent, Erhöhung des Treibstoffzolls (7.3.1993): 54,6 Prozent. Ganz allgemein gilt, dass bei Abstimmungen über kontroverse Vorlagen, z.B. in den Bereichen Verkehrs-, Ausländer- und Sicherheitspolitik, die Stimmbeteiligung in den letzten Jahren regelmäßig über 50 Prozent lag. Siehe hierzu auch Eschet-Schwarz (1989).

16. Siehe hierzu auch Kostede (1991).

17. Eine ähnliche Einschätzung lässt sich wiederum für die USA vornehmen. Lupia (1994) analysiert den Informationsstand der sich an der Abstimmung beteiligenden Stimmbürger bei fünf kalifornischen Versicherungsinitiativen. Er stellt fest, dass die Stimmbürger bei allen fünf Vorlagen herauszufinden versuchten, welche von der Versicherungsindustrie unterstützt 


\section{Gebhard Kirchgässner}

Geht man (zu Recht) davon aus, dass es erheblicher Sachkenntnisse bedarf, um politische Entscheide sachgerecht zu treffen, so könnte man die Auffassung vertreten, dass Entscheide in einer repräsentativen Demokratie im allgemeinen mit mehr Sachkenntnis getroffen werden als in einer direkten Demokratie. Schließlich haben die Abgeordneten größere Anreize, sich über politische Angelegenheiten zu informieren, als die einzelnen Bürger. Bei dieser Argumentation gerät man jedoch leicht in die Gefahr, den nicht immer überzeugenden tatsächlichen Zustand der direkten Demokratie mit den idealen Bedingungen einer repräsentativen Demokratie $\mathrm{zu}$ vergleichen. Tatsächlich sind die Abgeordneten eines Parlaments über die zur Abstimmung anliegenden Fragen häufig kaum informiert. Die Entscheidungen werden in Ausschüssen durch eine kleine Zahl Interessierter und Informierter getroffen, und die theoretisch allein ihrem Gewissen verantwortlichen Abgeordneten stimmen im Plenum, soweit sie überhaupt anwesend sind, entsprechend den Vorgaben ihrer Ausschussvertreter. Eine offene Diskussion findet dabei kaum statt. Zudem besteht ein starker Fraktionszwang: Abweichungen von der Partei- bzw. Fraktionslinie können mit erheblichen Sanktionen belegt werden; im schlimmsten Fall droht das Ende der politischen Karriere. Da außerdem in großen Parlamenten eine einzelne (abweichende) Stimme in aller Regel keinen Einfluss hat, haben viele Abgeordnete kaum Anreize, sich über Einzelheiten der zur Abstimmung stehenden Angelegenheiten zu informieren. Dies führt u.a. dazu, dass die Diskussionen im Plenum steril werden: Im Saal muss niemand überzeugt werden, da der Ausgang festliegt. Reden dienen vor allem der partei-internen Selbstdarstellung sowie - bei Übertragungen im Fernsehen - der Darstellung nach außen. Aber auch dort muss niemand von der Sache überzeugt werden; die Bürger haben ja keine Möglichkeit, auf die Angelegenheiten direkt Einfluss zu nehmen.

Dies führt dazu, dass über viele wichtige politische Entscheidungen in der Bevölkerung kaum Diskussionen geführt werden. So wurde z.B. die Frage, ob die Bundesrepublik Deutschland dem Vertrag von Maastricht beitreten soll, vor dem Nein der Dänen in der Bevölkerung kaum und auch danach nicht sehr ausführlich diskutiert. Immerhin hat die Bundesrepublik mit diesem Vertrag u.a. auf ihre Souveränität in der Geldpolitik verzichtet. Da es deutliche

\footnotetext{
wurde, um danach ihre eigene politische Präferenz mit derjenigen der Versicherungsindustrie zu vergleichen. Bowler und Donovan (1998) finden Evidenz dafür, dass uninformierte Wähler bei Sachabstimmungen eher nicht zur Abstimmung gehen, dass diejenigen, die zur Abstimmung gehen, Informationen entweder zu niedrigen Kosten gewinnen, wie etwa über Kommentare in Zeitungen, Fernsehwerbungen sowie Unterhaltungen mit Freunden und Nachbarn, oder sich anschauen, wie die Interessen von beteiligten politischen Eliten gelagert sind, um ihre eigenen Präferenzen in Relation zu denjenigen der politischen Eliten zu setzen. Gerber und Lupia (1996) stellen schließlich fest, dass die (Wahlkampf-) Ausgaben von Befürwortern und Gegnern einer Initiative im Abstimmungsprozess einen Einfluss auf den Informationsstand der Stimmbürger haben. Sind die Ausgaben beider sich einander gegenüberstehender Gruppen hoch, ist auch der Informationsstand der Bürger hoch. Werden keine Ausgaben oder nur einseitige Ausgaben getätigt, ist der Informationsstand der Bürger niedriger.
} 


\section{Wirtschaftliche Auswirkungen der direkten Demokratie}

Anzeichen dafür gab, dass dieser Vertrag in der deutschen Bevölkerung keine Mehrheit finden würde, hatten die Politiker, die nahezu geschlossen für dieses Vertragswerk eintraten, auch keinen Anreiz, hier eine Diskussion auszulösen.

Vergleicht man so die Verfassungswirklichkeit in der Bundesrepublik Deutschland mit jener in der Schweiz, kann man durchaus in Frage stellen, dass der durchschnittliche Bundestagsabgeordnete bei seiner Entscheidung besser informiert ist als der durchschnittliche schweizerische Stimmbürger. Soweit dies nicht der Fall ist, geht aber der wesentliche Vorteil der repräsentativen gegenüber der direkten Demokratie verloren, wobei die repräsentative Demokratie überdies den Nachteil einer schlechter informierten Öffentlichkeit hat. ${ }^{18}$ So aber sind die Schweizer Bürgerinnen und Bürger im Schnitt besser über die zur Entscheidung anstehenden politischen Entscheidungen informiert als die deutschen Bundesbürger. Der Grund dafür ist nicht in unterschiedlichen individuellen Einstellungen $\mathrm{zu}$ suchen, sondern darin, dass die Institutionen der direkten Demokratie den Stimmbürgern wesentliche zusätzliche Anreize vermitteln, sich über politische Angelegenheiten zu informieren.

Was für die einfachen Abgeordneten im Vergleich mit den Bürgern gilt, gilt selbstverständlich nicht für die Spezialisten der verschiedenen Parteien auf den entsprechenden Gebieten. Sie dürften im allgemeinen gut über die jeweiligen Probleme informiert sein, und sie sind tatsächlich in der Lage, überlegtere und informiertere Entscheidungen zu treffen als die Bevölkerung. Nun können diese Spezialisten - und sie tun dies auch - ihr Fachwissen bei dem einer Volksabstimmung vorangehenden parlamentarischen Entscheidungsprozess einbringen, und sie können sich außerdem im öffentlichen Diskurs vor der Volksabstimmung äußern. Insofern findet dieses Fachwissen auch in direkten Demokratien Berücksichtigung. Es stellt sich jedoch die Frage, ob diese Spezialisten in rein repräsentativen Systemen genügend Anreize haben, die (allgemeinen) Interessen der Bevölkerung zu berücksichtigen, oder ob sie nicht vielmehr häufig versuchen, ihre eigenen (partikulären) Interessen bzw. diejenigen ihrer spezifischen Klientel durchzusetzen. Schließlich sind sie oft Interessenvertreter bestimmter Gruppen, für die sie in den Parlamenten sitzen. Die moderne politische Ökonomie zeigt, dass solche Politiker Anreize haben, systematisch von den Interessen der Bevölkerung abzuweichen. Daher stellt sich die Frage, ob die wesentlich durch Spezialisten getroffenen „informierteren“ Entscheidungen in rein repräsentativen Demokratien wirklich "sachgemäßer“ sind als Entscheidungen in direkten Demokratien, wo die Vorschläge der Spezialisten auch die Zustimmung der Bevölkerung finden müssen.

18. Zum Vergleich der beiden politischen Systeme siehe auch Kirchgässner und Pommerehne (1992). 


\section{Die Effizienz der direkten Demokratie: Empirische Ergebnisse}

Gemäß dem, was in den Abschnitten zuvor ausgeführt wurde, haben die direkten Volksrechte in der Schweiz zwei Konsequenzen: Erstens führt der politische Diskurs, der einer Abstimmung vorangeht, dazu, dass die Bürgerinnen und Bürger im allgemeinen besser informiert sind als die Bürgerinnen und Bürger in einem rein repräsentativen System und möglicherweise sogar als deren Abgeordnete. Zweitens kann der Diskurs dazu führen, dass zumindest einige Bürgerinnen und Bürger sich Gedanken über ihre Präferenzen machen und sich auch verantwortlicher für ihr Gemeinwesen fühlen. Beides sollte Auswirkungen auf die wirtschaftspolitischen Entscheidungen und auch auf die wirtschaftlichen Ergebnisse haben.

Wie in der Einleitung bereits ausgeführt wurde, wird von den Gegnern der direkten Demokratie in der Schweiz, häufig behauptet, die direkte Demokratie behindere die Anpassungsfähigkeit der schweizerischen Politik an die sich ändernden internationalen Rahmenbedingungen und schränke damit die internationale Wettbewerbsfähigkeit der schweizerischen Wirtschaft ein. Als Evidenz dafür wird angegeben, dass die Schweiz in den neunziger Jahren die geringste Wachstumsrate aller OECD-Staaten hatte. Dem kann man jedoch entgegenhalten, dass die Schweiz nach wie vor ein sehr hohes Einkommensniveau und eine sehr niedrige Arbeitslosenquote aufweist. Zudem rangiert sie in den Ranglisten, die von verschiedenen Institutionen bezüglich der internationalen Wettbewerbsfähigkeit der Industriestaaten aufgestellt werden, regelmäßig weit oben.

Diese anekdotische Evidenz ist somit widersprüchlich und lässt keine eindeutigen Aussagen zu. Dafür bedarf es systematischer empirischer Studien. Solche Analysen gibt es heute für die Vereinigten Staaten und die Schweiz. Gerade die Schweiz eignet sich besonders für solche Untersuchungen, da die Volksrechte auf den Ebenen der Gemeinden und Kantone sehr unterschiedlich ausgebaut sind. So gibt es Gemeinden, in denen das Parlament über die Finanzangelegenheiten entscheidet, ohne dass die Bürgerinnen und Bürger direkte Einspruchsmöglichkeiten haben, während in anderen Gemeinden die Festlegung der Steuersätze dem obligatorischen oder fakultativen Referendum unterliegt. Insofern gibt es Gemeinden (und Kantone) mit einem rein repräsentativen Entscheidungsverfahren mit Bezug auf Finanzfragen und solche mit (unterschiedlich ausgebildeten) direkten Volksrechten. ${ }^{19}$ Die

19. Gewisse Elemente direkter Demokratie gibt es in allen schweizerischen Gemeinden und Kantonen. Insbesondere werden überall die Gemeinde- bzw. Kantonsregierungen direkt vom Volk bestimmt. Dies ist jedoch für die hier geführte Diskussion ohne Belang. Wichtig ist, dass bezüglich der Möglichkeiten, auf Sachentscheidungen Einfluss zu nehmen, erhebliche Unterschiede bestehen. (Zudem ist umstritten, ob die direkte Wahl von Regierungsmitgliedern oder Präsidenten überhaupt als Teil der direkten Demokratie betrachtet werden soll, da es dabei ja auch um Delegation und nicht um direkte Entscheidung der Bürgerinnen und Bürger geht. Siehe hierzu Marschall (1997).) 


\section{Wirtschaftliche Auswirkungen der direkten Demokratie}

Schweiz ist daher gleichsam ein „Labor“, mit dessen Hilfe man die Auswirkungen der direkten Demokratie studieren kann. Ähnliches gilt, wenn auch in abgeschwächtem Maße, für die Vereinigten Staaten.

Die erste systematische empirische Arbeit über die wirtschaftspolitischen Auswirkungen der direkten Demokratie in der Schweiz stammt von Pommerehne (1978). Mit Daten der 110 größten Schweizer Städte hat er für den Durchschnitt der Jahre 1968 bis 1972 die Auswirkungen der direkten Volksrechte auf den Budgetierungsprozess in diesen Städten untersucht. Dabei zeigte sich, dass in Gemeinden mit direkten Volksrechten bezüglich Finanzfragen die öffentlichen Ausgaben eher den Präferenzen des Medianwählers entsprechen als in Gemeinden, die keine solchen Rechte kennen. ${ }^{20}$ Für die gleichen Städte haben Schneider und Pommerehne (1983) den Anstieg der Staatsausgaben im Zeitraum von 1965 bis 1975 untersucht. Dabei zeigte sich, dass dieser Anstieg in den Gemeinden mit direkter Demokratie in Finanzfragen - ceteris paribus - um knapp drei Prozent unter dem Anstieg in den übrigen Gemeinden lag. Tatsächlich betrug der Anstieg der Staatsausgaben in den Gemeinden mit repräsentativem System durchschnittlich 9,6 Prozent. Eine Simulation ergab, dass er nur 6,8 Prozent betragen hätte, wenn in diesen Gemeinden die entsprechenden direkten Volksrechte existiert hätten. Bei diesen Untersuchungen wurden Gemeinden als 'direkt demokratisch' betrachtet, wenn in ihrer Verfassung vorgesehen war, dass die Bürgerinnen und Bürger mit Hilfe eines fakultativen oder obligatorischen Referendums oder in einer Gemeindeversammlung die Möglichkeit hatten, auf die Steuersätze, den Budgetvorschlag sowie das geplante Defizit Einfluss zu nehmen bzw. die entsprechenden Vorlagen der Gemeinderegierung bzw. -verwaltung abzulehnen.

Ähnliche Resultate findet Matsusaka (1995) für die 49 Bundesstaaten der USA über den Zeitraum von 1960 bis $1990 .^{21}$ Zwar sind in den Bundesstaaten, in welchen die Bürger direkte Eingriffsrechte haben, die Ausgaben auf lokaler Ebene signifikant höher als in Staaten, in denen diese Möglichkeiten nicht gegeben sind. Dies wird aber überkompensiert durch Reduktionen auf der Ebene der Bundesstaaten, da staatliche Aufgaben dann eher auf lokaler Ebene und damit näher am Bürger ausgeführt werden. Dabei ist das gesamte Ausgabenniveau um so geringer, je geringer die Stimmenzahl ist, die zum Ergreifen einer Initiative notwendig ist. Bei einem Stimmenerfordernis von 10

20. E.R. Gerber $(1996,1999)$ findet gleichgerichtete Evidenz für die USA.

21. Zuvor hatte allerdings Farnham (1990) in einer Querschnittsuntersuchung der Staatsausgaben von 735 amerikanischen Städten für die Jahre 1981/82 nur geringfügige oder gegenläufige (in beiden Fällen nur teilweise signifikante) Effekte festgestellt. Die von Zax (1989) vorgestellten Ergebnisse sind ebenfalls gegenläufig und zeigen höhere Staatsausgaben pro Kopf für 50 U.S. Bundesstaaten und 1305 amerikanische Städte und Gemeinden im Jahre 1980. Die Ergebnisse von Matsusaka (1995) sind, da sie auf einer Paneldatenanalyse beruhen, jedoch umfassender und daher überzeugender. Zudem wurden seine Ergebnisse von Rueben (2000) in einer weiteren Paneldatenanalyse für die U.S.-Bundesstaaten mit veränderter Stichprobe weitgehend bestätigt. 


\section{Gebhard Kirchgässner}

Prozent reduzieren sich die gesamten Ausgaben dieser beiden Ebenen um etwa 55 U.S.-Dollar pro Kopf und Jahr, bei einem Stimmenerfordernis von 2 Prozent dagegen um etwa 110 U.S.-Dollar. Übersteigt das Erfordernis 10 Prozent, so tritt keine signifikante Ausgabenreduktion mehr ein. Bei dem häufigsten Stimmenerfordernis von 5 Prozent reduzieren sich die Ausgaben um etwa 90 U.S.-Dollar pro Kopf und Jahr, was bei einem jährlichen Durchschnitt dieser Ausgaben von 2,300 U.S.-Dollar pro Kopf für Staaten mit Initiativen eine Verringerung dieser Ausgaben um ca. 4 Prozent bedeutet. Außerdem finanzieren sich, wie Matsusaka feststellt, amerikanische Bundesstaaten mit Initiativrecht stärker über Gebühren und Beiträge als über aufkommenselastische Steuern.

Direkt-demokratisch organisierte Gebietskörperschaften haben auch eine niedrigere Staatsverschuldung. Kiewiet und Szakaly (1996) zeigen, dass von 1961 bis 1990 der pro Kopf Schuldenstand der U.S.-Bundesstaaten, in denen die Nettoneuverschuldung ein Referendum passieren musste, mit 166 U.S.-Dollar im Durchschnitt um ein Drittel niedriger war als im Durchschnitt aller Bundesstaaten (267 U.S.-Dollar), ohne dass deshalb Staatsaufgaben auf die lokale Ebene verdrängt wurden. ${ }^{22}$ Bohn und Inman (1996) weisen darauf hin, dass formale fiskalische Beschränkungen in den U.S.-Bundesstaaten vor allem dann erfolgreich waren, wenn sie vom Verfassungsgericht des jeweiligen Bundesstaates kontrolliert wurden. Diejenigen Verfassungsgerichte, deren Richter von den Bürgern gewählt wurden, setzten formale fiskalische Beschränkungen konsequenter durch: In den 21 Bundesstaaten, in denen die Wahl der Richter durch das Volk stattfand, waren die Haushaltsdefizite durchschnittlich um 96 U.S.Dollar pro Bürger niedriger als in den 15 Bundesstaaten, in denen die Richter von der Legislative oder Exekutive ernannt wurden.

Feld und Kirchgässner (1999) haben mit Hilfe eines simultanen Gleichungsmodells verschiedene Aspekte staatlicher Haushaltspolitik untersucht. Für die 131 größten schweizerischen Städte im Jahr 1990 zeigen sie, dass der Bruttoschuldenstand pro Kopf - ceteris paribus - in den repräsentativdemokratischen Städten um ca. 10,000 SFr und damit um 45 Prozent niedriger gewesen wäre, wenn diese direkt-demokratisch organisiert gewesen wären. ${ }^{23}$ Zudem hatten Städte mit Budgetreferendum um 14 Prozent niedrigere Staatsausgaben, eine um 5 Prozent höhere Selbstfinanzierungsquote und einen um 14 Prozent höheren Mediansteuersatz. Dies spricht dafür, dass die Bürger in diesen Gebietskörperschaften zwar weniger öffentliche Leistungen nachfragen,

22. McEachern (1978) stellt dagegen fest, dass die lokale Staatsschuld pro Kopf in 50 U.S.Bundesstaaten nicht signifikant niedriger ist, wenn die Bürger in einem Referendum darüber entscheiden können. Ist jedoch für eine Erhöhung der Staatsschuld ein Referendum mit höherem Mehrheitserfordernis als der einfachen Mehrheit notwendig, sinkt die Staatsschuld um 132 U.S. Dollar pro Kopf.

23. Schließt man die Stadt Zürich, die mit ihrem extrem hohen Schuldenstand nach allen statistischen Kriterien einen Ausreißer darstellt, aus, so beträgt die simulierte Differenz immer noch 4.500 SFr bzw. 24 Prozent. Wie in dieser Studie außerdem gezeigt wurde, führen weder formale fiskalische Beschränkungen noch ein sehr einflussreicher Bürgermeister oder Finanzsekretär zu einer so starken Eindämmung der Staatsschuld. 


\section{Wirtschaftliche Auswirkungen der direkten Demokratie}

für diese Leistungen aber höhere und wahrscheinlich adäquatere Preise zu zahlen bereit sind als in rein parlamentarischen Systemen, woraus eine geringere öffentliche Verschuldung resultiert. Somit kommt die Referendumsdemokratie der Wicksell'schen Vorstellung einer Verknüpfung von Steuerpreis und öffentlicher Leistung näher als die rein parlamentarische Demokratie.

Die Frage ist freilich, ob dieses niedrigere Ausgabenniveau - gemessen an den Präferenzen der Bürger - auch effizient ist. Niedrigere Ausgaben könnten auch, wie Breton (1996) vermutet, Ausdruck eines ungenügenden Stimmentauschs sein, so dass Referenden und Initiativen zu ineffizienten Lösungen führten. Hierzu gesicherte Aussagen $\mathrm{zu}$ machen, ist außerordentlich schwierig. Entsprechend gibt es auch kaum Studien, in welchen dies versucht wird. ${ }^{24}$ Indirekte Evidenz für höhere staatliche Effizienz in direkten Demokratien findet sich jedoch in einer Studie über die Steuermoral. Pommerehne und Weck-Hannemann (1996) zeigen, dass diese in Kantonen mit direkter Demokratie in Finanzfragen höher ist als in solchen mit in Bezug auf diese Frage rein repräsentativen Entscheidungen. So stellen sie (für die Jahre 1965, 1970 und 1978) fest, dass Steuern in den Schweizer Kantonen, in denen Bürger weitgehend über das Budget mitentscheiden, in geringerem Ausmaß hinterzogen wurden: Gegenüber dem Durchschnitt der Kantone wurde pro Steuerpflichtigem und Jahr - ceteris paribus - rund 1.500 SFr weniger Einkommen verheimlicht. ${ }^{25}$ Wenn aber, was anzunehmen ist, die Bereitschaft, Steuern zu zahlen, um so höher ist, je zufriedener die Bürger mit den ihnen gebotenen öffentlichen Leistungen sind, dann spricht dieses Ergebnis für höhere Zufriedenheit und damit auch für höhere Effizienz der staatlich angebotenen Leistungen in direkt-demokratischen Systemen.

Pommerehne (1983) hat untersucht, ob es Kosten- und Preisunterschiede bei der Müllabfuhr gibt, die auf die unterschiedliche Organisation der Müllabfuhr zurückzuführen sind. Hierzu hat er für das Jahr 1970 deren Kosten in den 103 größten schweizerischen Städten analysiert. Dabei stellte er fest, dass die durchschnittlichen Abfuhrkosten (pro Haushalt) in Städten mit direkter Demokratie und privater Müllabfuhr - ceteris paribus - am niedrigsten sind. Die durchschnittlichen Abfuhrkosten sind um rund 10 Prozent höher, wenn es anstelle der privaten eine städtische Müllabfuhr gibt. In Städten mit repräsentativer Demokratie liegen die Kosten der privaten Müllabfuhr um etwas mehr als 20 Prozent höher als in direkten Demokratien. Die durchschnittlichen Abfuhrkosten sind am höchsten in Städten mit repräsentativer Demokratie und öffentlicher Müllabfuhr; dort sind sie um 30 Prozent höher als in Städten mit direkter Demokratie und privater Müllabfuhr.

24. Eine Ausnahme hierzu bildet Noam (1980). Das dort benutzte Effizienzmaß ist aber wenig operativ.

25. Es gibt auch theoretische Argumente dafür, dass die Bürger in direkten Demokratien weniger Steuern hinterziehen als in rein repräsentativen Systemen. Siehe hierzu Pommerehne, Hart und Feld (1997). 


\section{Gebhard Kirchgässner}

Feld und Savioz (1997) untersuchen den Zusammenhang zwischen Fiskalreferenden und der Wirtschaftsleistung der Schweizer Kantone, gemessen an ihrem Bruttoinlandsprodukt. Mit jährlichen Daten über den Zeitraum von 1984 bis 1993 kommen sie zu dem Ergebnis, dass die Kantone mit Fiskalreferenden - ceteris paribus - ein um etwa 5 Prozent höheres Bruttoinlandsprodukt pro Kopf erwirtschafteten als die übrigen Kantone. ${ }^{26}$ In dieser Arbeit wird auch untersucht, ob die Kausalität möglicherweise in die umgekehrte Richtung geht: dass sich die reicheren Kantone mehr direkte Demokratie leisten können. Die empirische Evidenz spricht jedoch dagegen. Zudem wird der Einfluss der direkten Demokratie kaum geschmälert, wenn weitere Einflussfaktoren berücksichtigt werden.

Fasst man alle diese Ergebnisse zusammen, so ergibt sich, dass die direkte Demokratie in der Schweiz zu einer Ausgabenpolitik führt, die näher an den Präferenzen der Bürgerinnen und Bürger ist, und die darüber hinaus mit einer geringeren Staatsschuld, einer effizienteren Verwaltung und höherem Wohlstand verbunden ist. Dies bewirkt eine stärkere Identifikation mit dem Gemeinwesen; und so ist es auch nicht verwunderlich, dass Frey und Stutzer (2000) feststellen, dass die Bürgerinnen und Bürger in Gemeinden mit stärker ausgeprägten direkten Volksrechten zufriedener sind. Die vergleichbaren Studien für die Vereinigten Staaten sprechen insgesamt zwar auch für die direkte Demokratie, ihre Ergebnisse sind jedoch weniger eindeutig als diejenigen für die Schweiz. Ein Grund dafür könnte in den unterschiedlichen institutionellen Ausgestaltungen liegen. So stimmen z.B. in Kalifornien die Stimmbürger nur einmal im Jahr ab. Dann kommen aber bis zu vierzig oder mehr Vorlagen zusammen. Damit kann der Diskussionsprozess, der einer Abstimmung vorangehen sollte, überhaupt nicht - oder jedenfalls nicht im erforderlichen Ausmaß - stattfinden.

Von denjenigen, welche die direkte Demokratie in der Schweiz auf Bundesebene einschränken oder gar abschaffen möchten, wird bezweifelt, ob die Ergebnisse, die für die Kantone und Gemeinden erzielt wurden, auf die Bundesebene übertragbar sind. ${ }^{27}$ Nun wäre es naiv abzustreiten, dass eine Übertragung empirischer Ergebnisse von einer föderalen Ebene auf eine andere Probleme aufwerfen könnte. Theoretische Gründe dafür können sicher gefunden werden. Zudem gibt es keine Möglichkeit, hierüber empirische Untersuchungen anzustellen, um diese Hypothese zu verwerfen. Man kann daher die Möglichkeit, dass die Auswirkungen der direkten Demokratie auf der Ebene des Bundes völlig anders sind als auf den Ebenen der Kantone und Gemeinden, nicht a priori ausschließen. Was aber spricht für diese Behauptung?

Häufig wird die angebliche Reformunfähigkeit des politischen Systems der Schweiz auf das fakultative Referendum und seinen Missbrauch durch

26. Bei einer Querschnittsanalyse für das Jahr 1990 ergab sich sogar ein Unterschied von 15 Prozent.

27. Siehe z.B. Borner in Borner und Rentsch (1997, S. 109f.). 


\section{Wirtschaftliche Auswirkungen der direkten Demokratie}

Interessengruppen zurückgeführt. ${ }^{28}$ Man könnte sich daher vorstellen, dass dem fakultativen Referendum auf der Bundesebene eine andere Rolle zukommt als auf den unteren Ebenen, und dass es auf Bundesebene eher „missbraucht" wird. Aber welche Unterschiede bestehen hier und wie kann es zu einem stärkeren Missbrauch kommen? Da die Volksrechte in den Kantonen und Gemeinden teilweise erheblich stärker ausgeprägt sind als auf Bundesebene und da insbesondere die Kosten, ein fakultatives Referendum zu ergreifen (soweit dies überhaupt möglich ist), viel geringer sind, ist ein solcher „Missbrauch" der Volksrechte eher auf den unteren Ebenen zu erwarten als auf Bundesebene. Warum aber sollen dann die Ergebnisse der direkten Demokratie auf den unteren Ebenen eher akzeptabel sein?

Betrachtet man die vorgestellten empirischen Ergebnisse über die wirtschaftlichen Auswirkungen der direkten Demokratie genauer, dann spricht viel dafür, dass Probleme u.a. dadurch entstehen, dass auf Bundesebene Volksrechte fehlen, die in vielen Kantonen und Gemeinden vorhanden sind. Falls die Ausgaben des Bundes heute tatsächlich zu hoch sein sollten, mag das damit zusammen hängen, dass dieser bisher kein Finanzreferendum kennt, welches zu einer Beschränkung der Ausgaben beitragen könnte. Und der Anstieg der eidgenössischen Schuld wäre möglicherweise geringer ausgefallen, wenn man die Stimmbürgerinnen und Stimmbürger um ihre Zustimmung zu den (geplanten) Defiziten hätte fragen müssen. Hier scheint eher das Fehlen als ein Übermaß an direkten Volksrechten zu den Problemen beigetragen zu haben. Auf jeden Fall spricht kaum etwas dafür, dass das fakultative Referendum auf den verschiedenen föderalen Ebenen vollkommen unterschiedliche Auswirkungen hat.

Man kann schließlich argumentieren, dass die direkte Demokratie auf den unteren föderalen Ebenen deshalb besser funktioniert, weil die Bürgerinnen und Bürger dort direkter mit den anstehenden Problemen vertraut sind und deshalb auch eher Entscheidungskompetenz haben. Dieses Argument ist sicher nicht einfach von der Hand zu weisen, und es gilt insbesondere für die lokale Ebene. Man kann das gleiche Argument aber auch auf die Parlamentarier anwenden. Letztlich geht es wieder darum, wieweit angesichts der Komplexität der zu lösenden Probleme Delegation politischer Entscheidungen an Spezialisten notwendig ist. Damit sind wir wieder beim Informationsproblem der Demokratie. Entsprechend den oben vorgebrachten Argumenten dürften jedoch aus der Perspektive der Informationsverarbeitung die politischen Entscheidungen in der direkten Demokratie nicht schlechter sein als in rein repräsentativen Systemen. Dies gilt für alle föderalen Ebenen. Insofern lässt sich auch daraus, dass die Bürgerinnen und Bürger auf Kantons- und Gemeindeebene näher an den zur Entscheidung anstehenden Problemen sind, kein grundsätzliches Argument gegen die Übertragbarkeit der vorgelegten empirischen Ergebnisse auf die Bundesebene ableiten.

28. Siehe z.B. Borner, Brunetti und Straubhaar (1994, S. 25ff., S. 124ff.). 


\section{Abschließende Bemerkungen: Beliebte Gegenargumente und die Erfahrung von Weimar}

Ein organisatorisches Argument, welches gegen die Einführung der direkten Demokratie in der Bundesrepublik Deutschland häufig ins Feld geführt wird, ist die Größe des Gemeinwesens: In der kleinen Schweiz mit ihren im Vergleich zu den Gliedstaaten anderer Länder kleinen Kantonen könne ein solches System zwar funktionieren, in der sehr viel größeren Bundesrepublik Deutschland jedoch nicht. Dieses Argument negiert zum einen die amerikanische Erfahrung: der Bundesstaat Kalifornien mit seiner stark ausgeprägten direkten Demokratie hat immerhin 32,3 Millionen Einwohner und eine Fläche von 411.500 Quadratkilometern, was 115 Prozent der Fläche der Bundesrepublik Deutschland entspricht. Zudem wird dieses Argument mit zunehmender Technisierung und Entwicklung der Informationsmedien immer weniger überzeugend. Durch die neuen Medien sinken die Kosten für die Durchführung von Initiativen und Referenden ständig: Die praktischen Möglichkeiten für Abstimmungen sind auch in größeren Staaten heute mehr denn je gegeben. ${ }^{29}$

In die gleiche Richtung zielt die Behauptung, die direkte Demokratie könne aufgrund der besonderen Tradition und Kultur zwar in der Schweiz funktionieren, aber nicht in Deutschland (oder in Gesamteuropa), wo eine solche Tradition fehle. An diesem Argument ist richtig, dass in der Schweiz eine besondere Kultur des politischen Diskurses besteht, die sich z.B. deutlich von jener in der Bundesrepublik Deutschland unterscheidet. Diese Kultur ist aber nicht nur eine Voraussetzung, sondern auch eine Folge der direkten Demokratie: Sie kann sich überhaupt nur dann entwickeln, wenn die Bürgerinnen und Bürger auch die Möglichkeit haben, über Sachfragen abzustimmen. Das Fehlen einer solchen Kultur bzw. Tradition kann daher kein Argument gegen die Einführung der direkten Demokratie sein, es mag als Argument dafür dienen, diese schrittweise und beginnend mit den unteren staatlichen Ebenen einzuführen. So könnte sich eine solche Kultur auch in Deutschland entwickeln.

Schließlich werden in Deutschland häufig noch die schlechten Erfahrungen von Weimar als Argument gegen die direkte Demokratie in Stellung gebracht: Die Weimarer Republik sei, so wird behauptet, unter anderem an der direkten Demokratie gescheitert, und die Mütter und Väter des Grundgesetzes hätten darum keine direkten Volksrechte auf Bundesebene in die Verfassung aufgenommen. $^{30}$ Die neuere Forschung hat jedoch gezeigt, dass diese Behauptung in all ihren Teilen so nicht aufrecht erhalten werden kann. ${ }^{31}$ Zum einen haben die direkten Volksrechte in der Republik von Weimar nur eine untergeordnete Rolle gespielt: Auf Reichsebene kamen nur drei Volksbegehren über das Vorverfahren hinaus in das Stadium des Sammelns von

29. Siehe hierzu auch Budge (1996).

30. Siehe hierzu die zahlreichen Verweise bei Obst (1986, S. 121, Fn. 1).

31. Kirchgässner, Feld und Savioz (1999, S. 145ff.) sowie insbesondere Jung $(1989,1993)$. 


\section{Wirtschaftliche Auswirkungen der direkten Demokratie}

Unterschriften, und nur über zwei wurde tatsächlich abgestimmt, wobei in beiden Fällen die Regierung gewann. Es ist zwar richtig, dass die Rechtsparteien das Volksbegehren gegen den Young-Plan, über welchen am 22. Dezember 1929 abgestimmt wurde, zu Angriffen gegen den demokratischen Staat nutzten, was möglicherweise den gegen Ende der Weimarer Republik wieder zunehmenden Druck der Straße zusätzlich verstärkte, und dass dieser erheblichen Einfluss auf die Politik ausübte und den Übergang zum Nationalsozialismus wesentlich mitbewirkte. ${ }^{32}$ Dennoch spricht alles dafür, dass, wie Bugiel bereits 1991 schrieb, „die Weimarer Republik eher an der mangelnden Funktion des Parlamentarismus als an dem extensiven Gebrauch direktdemokratischer Mitwirkungsmöglichkeiten gescheitert ist.“33

Zweitens wird in diesem Zusammenhang häufig die Direktwahl des Reichspräsidenten ins Feld geführt. Die direkte Wahl von Regierungsmitgliedern durch das Volk, wie sie in der Bundesrepublik Deutschland bei der Wahl des Oberbürgermeisters auf Gemeindeebene in vielen Fällen möglich ist, hat mit direkten Entscheidungen der Stimmbürger über Sachfragen nichts $\mathrm{zu}$ tun. Es ist richtig, dass die Wahl von Paul von Hindenburg zum Reichspräsidenten im Jahr 1925 die Demokratie in Weimar nicht gerade gestärkt hat und dass die Kandidatur von Adolf Hitler zu diesem Amt im Jahre 1932 diesem die Möglichkeit der Profilierung gegeben hat. Aber all dies spricht nicht gegen die direkte Mitwirkung des Volkes bei Sachentscheidungen. Allerdings legt es beim Vorschlag der Direktwahl des Bundespräsidenten Vorsicht nahe. Nicht umsonst werden ja in der Schweiz zwar die Mitglieder aller Kantons- und Gemeinderegierungen, aber nicht jene der Bundesregierung, direkt vom Volk gewählt.

Schließlich ist zur Kenntnis zu nehmen, dass bei der Diskussion um die neue deutsche Verfassung nach dem Zweiten Weltkrieg das Argument der schlechten Erfahrungen von Weimar nur eine untergeordnete Rolle spielte. Viel wichtiger war ein generelles Misstrauen gegenüber dem Volk sowie die Angst, die radikale Linke könnte sich dieses Instrument zunutze machen. ${ }^{34}$ Aus Angst vor dieser hat man schließlich auch auf ein „Gründungsplebiszit" verzichtet, obwohl dies nach allgemeiner Auffassung auch der Alliierten zunächst vorgesehen war. Wie immer aber man die Gründe und Motive, welche die Mütter und Väter des Grundgesetzes im Parlamentarischen Rat bewogen haben, den Bürgerinnen und Bürgern auf Bundesebene direkte Volksrechte zu verweigern, vor dem Hintergrund der damaligen Situation auch beurteilen mag, nach einer insgesamt doch recht erfolgreichen Entwicklung der deutschen Demokratie in den letzten 50 Jahren kann man sie kaum mehr aufrecht erhalten. Selbst wenn man zugestehen wollte, dass die Entscheidung gegen die direkte

32. Eine dezidiert andere Position vertritt in diesem Zusammenhang Jung (1989, S. 128ff.; 1989a). Er bestreitet, dass die in diesem Abstimmungskampf erfolgte Mobilisierung und Profilierung der Rechten wesentlich zum Aufstieg des Nationalsozialismus beigetragen habe.

33. Bugiel (1991, S. 181). Siehe hierzu auch Pestalozza (1981, S. 29) sowie Obst (1986, S. 101ff.). 34. Siehe hierzu Jung (1994). 


\section{Gebhard Kirchgässner}

Demokratie in der Situation des Jahres 1949 „richtig“ war, so sind die Gründe, die damals zu dieser Entscheidung führten, heute fast vollständig entfallen.

Weder die tatsächliche Entwicklung in der Weimarer Republik noch die Gründe, welche die Mütter und Väter des Grundgesetzes dazu bewogen haben, mit der einzigen Ausnahme in Art. 29 Abs. 2 für den Fall der Neugliederung des Bundesgebietes keine direkten Volksrechte in die neue deutsche Verfassung aufzunehmen, sind daher hinreichend für die Ablehnung einer solchen Aufnahme zum heutigen Zeitpunkt.

\section{$\underline{\text { Literaturverzeichnis }}$}

Bohn, H. und R.P. Inman (1996), Balanced-Budget Rules and Public Deficits: Evidence from the U.S. States, Carnegie-Rochester Conference Series on Public Policy 45, 13-76.

Borner, S., und H. Rentsch (Hrsg) (1997), Wieviel direkte Demokratie verträgt die Schweiz? Rüegger, Chur/Zürich.

Borner, S., A. Brunetti und Th. Straubhaar (1990), Schweiz AG: Vom Sonderfall zum Sanierungsfall? Verlag Neue Zürcher Zeitung, Zürich.

Borner, S., A. Brunetti und Th. Straubhaar (1994), Die Schweiz im Alleingang. Verlag Neue Zürcher Zeitung, Zürich.

Bowler, S., und T. Donovan (1998), Demanding Choices: Opinion, Voting, and Direct Democracy. University of Michigan Press, Ann Arbor.

Breton, A. (1996), Competitive Governments: An Economic Theory of Politics and Public Finance. Cambridge University Press, Cambridge (England).

Budge, I. (1996), The New Challenge of Direct Democracy. Polity Press, Cambridge (England).

Bugiel, K. (1991), Volkswille und repräsentative Entscheidung: Zulässigkeit und Zweckmässigkeit von Volksabstimmungen nach dem Grundgesetz. Nomos, Baden-Baden.

Downs, A. (1957), An Economic Theory of Democracy. New York; deutsche Übersetzung: Ökonomische Theorie der Demokratie. Mohr (Siebeck), Tübingen 1968.

Eschet-Schwarz, A. (1989), La démocratie semi directe en Suisse: entre la théorie et la réalité: 1879-1987, Canadian Journal of Political Science 22, 739-764.

Farnham, P.G. (1990), The Impact of Citizen Influence on Local Government Expenditure, Public Choice 64, 201-212.

Feld, L.P., und G. Kirchgässner (1999), Public Debt and Budgetary Procedures: Top Down or Bottom Up? Some Evidence from Swiss Municipalities, in: J.M. Poterba und J. von Hagen (Hrsg), Fiscal Institutions and Fiscal Performance. Chicago University Press und NBER, Chicago, 151-179.

Feld, L.P., und M.R. Savioz (1997), Direct Democracy Matters for Economic Performance: An Empirical Investigation, Kyklos 50, 507-538.

Frey, B.S., und G. Kirchgässner (1993), Volksabstimmungen, politische Ökonomie und Diskursethik, Analyse und Kritik 15, 129-149.

Frey, B.S., und A. Stutzer (2000), Happiness, Economy and Institutions, erscheint in: German Economic Review 1.

Gerber, E.R. (1996), Legislative Response to the Threat of Initiatives, American Journal of Political Science 40, 99-128.

Gerber, E.R. (1999), The Populist Paradox: Interest Group Influence and the Promise of Direct 


\section{Wirtschaftliche Auswirkungen der direkten Demokratie}

Legislation. Princeton University Press, Princeton.

Gerber, E.R. und A. Lupia (1996), Term Limits, Responsiveness and the Failures of Increased Competition, in: B. Grofman (Hrsg.), Legislative Term Limits: Public Choice Perspectives. Kluwer, Boston et al., 87-99.

Heussner, H.K. (1999), Ein Jahrhundert Volksgesetzgebung in den USA, in: H.K. Heussner und O. Jung (Hrsg), Mehr direkte Demokratie wagen. Olzog, München, 101122.

Jung, O. (1989), Direkte Demokratie in der Weimarer Republik: Die Fälle 'Aufwertung', 'Fürstenenteignung', 'Panzerkreuzerverbot' und 'Young-Plan'. Campus, Frankfurt/New York.

Jung, O. (1993), Die 'Weimarer Erfahrung' mit der Volksgesetzgebung: Kritik und Tragweite, Jahrbuch für Politik 3, 63-92.

Jung, O. (1994), Grundgesetz und Volksentscheid. Westdeutscher Verlag, Opladen.

Jung, O. (1998), Die Praxis direkter Demokratie unter den neuen Landesverfassungen, Zeitschrift für Gesetzgebung 13, 295-328.

Jürgens, G. (1993), Direkte Demokratie in den Bundesländern: Gemeinsamkeiten, Unterschiede, Erfahrungen, Vorbildfunktion für den Bund? Richard Boorberg Verlag, Stuttgart et al.

Kiewiet, D.R., und K. Szakaly (1996), Constitutional Limitations on Borrowing: An Analysis of State Bonded Indebtedness, Journal of Law, Economics and Organization 12, 62-97.

Kirchgässner, G. (1986), Economic Conditions and the Popularity of West German Parties: A Survey, European Journal on Political Research 14, 421-439.

Kirchgässner, G. (1990), Hebt ein knapper Wahlausgang die Wahlbeteiligung?, Eine Überprüfung der ökonomischen Theorie der Wahlbeteiligung anhand der Bundestagswahl 1987, in: M. Kaase und H.-D. Klingemann (Hrsg.), Wahlen und Wähler: Analysen aus Anlaß der Bundestagswahl 1987. Westdeutscher Verlag, Opladen, 445-477.

Kirchgässner, G., und W.W. Pommerehne (1992), Zwischen Parteien- und Bundesstaat: Staatshandeln in der Schweiz und in der Bundesrepublik Deutschland, in: $H$. Abromeit und W.W. Pommerehne (Hrsg), Staatstätigkeit in der Schweiz. Haupt, Bern/ Stuttgart, 221-245.

Kirchgässner, G., L.P. Feld und M.R. Savioz (1999), Die direkte Demokratie: Modern, erfolgreich, entwicklungs- und exportfähig. Helbung und Lichtenhahn/Vahlen, Basel/ München.

Kleinewefers, H. (1997), Die direkten Volksrechte in der Schweiz aus ökonomischer Sicht, in: S. Borner und H. Rentsch (1997), 61-92, 343-346.

Kostede, N. (1991), Das Plebiszit als Lernprozess, DIE ZEIT Nr. 15 vom 5. April 1991, 5.

Lupia, A. (1994), Shortcuts Versus Encyclopaedias: Information and Voting Behavior in California Insurance Reform Elections, American Political Science Review 88, 63-76.

Lupia, A., und M.D. McCubbins (1998), The Democratic Dilemma: Can Citizens Learn What They Need to Know? Cambridge University Press, Cambridge (England).

Luthardt, W., und A. Waschkuhn (1997), Plebiszitäre Komponenten in der repräsentativen Demokratie: Entwicklungsstand und Perspektiven, in: A. Klein und R. Schmalz-Bruns (Hrsg), Politische Beteiligung und Bürgerengagement in Deutschland: Möglichkeiten und Grenzen. Nomos, Baden-Baden, 59-87.

McEachern, W.A. (1978), Collective Decision Rules and Local Debt Choice: A Test of the Median Voter Hypothesis, National Tax Journal 31, 129-136.

Marschall, St. (1997), Ist das unmittelbare Personenvotum ein "direktdemokratisches" 


\section{Gebhard Kirchgässner}

Verfahren?, Zeitschrift für Politikwissenschaft 7, 845-862.

Matsusaka, J.G. (1992), Economics of Direct Legislation, Quarterly Journal of Economics 107, 541-571.

Matsusaka, J.G. (1995), Fiscal Effects of the Voter Initiative: Evidence from the Last 30 Years, Journal of Political Economy 103, 587-623.

Nannestad, P., und M. Paldam (1994), The VP-Function: A Survey of the Literature on Vote and Popularity Functions After 25 Years, Public Choice 79, 213-245.

Noam, E.M. (1980), The Efficiency of Direct Democracy, Journal of Political Economy 88, 803-810.

Obst, C.-H., (1986), Chancen direkter Demokratie in der Bundesrepublik Deutschland: Zulässigkeit und politische Konsequenzen. Theurer, Köln.

Pestalozza, Ch. v. (1981), Der Popularvorbehalt: Direkte Demokratie in Deutschland. De Gruyter, Berlin.

Pommerehne, W.W. (1978), Institutional Approaches to Public Expenditure: Empirical Evidence from Swiss Municipalities, Journal of Public Economics 9, 255-280.

Pommerehne, W.W. (1983), Private versus öffentliche Müllabfuhr nochmals betrachtet, Finanzarchiv 41, 466-475.

Pommerehne, W.W., und H. Weck-Hannemann (1996), Tax Rates, Tax Administration and Income Tax Evasion in Switzerland, Public Choice 88, 161-170.

Pommerehne, W.W., A. Hart und L.P. Feld (1997), Steuerhinterziehung und ihre Kontrolle in unterschiedlichen politischen Systemen, Homo oeconomicus 14, 469-487.

Rueben, K. (2000), Tax Limitations and Government Growth: The Effect of State Tax and Expenditure Limits on State and Local Government, erscheint in: Journal of Political Economy 108.

Schmack-Reschke, T. (1997), Bürgerbeteiligung und Plebiszite in der Debatte der Gemeinsamen Verfassungskommission, in: N. Konegen und P. Nitschke (Hrsg.), Revision des Grundgesetzes? Ergebnisse der gemeinsamen Verfassungskommission (GVK) des Deutschen Bundestages und des Bundesrates. Leske und Budrich, Opladen, 77-105.

Schneider, F. (1985), Der Einfluss von Interessengruppen auf die Wirtschaftspolitik. Bern/ Stuttgart.

Schneider, F., und W.W. Pommerehne (1983), Macroeconomia della crescità in disequilibrio e settore pubblico in espansione: il peso delle differenze istituzionali, Rivista Internazionale di Scienze Economiche e Commerciali 33, 306-420.

Wittmann, W. (1998), Die Schweiz: Ende eines Mythos. Wirtschaftsverlag Langen Müller/ Herbig, München.

Zax, J.S. (1989), Initiatives and Government Expenditures, Public Choice 63, 267-277.

Abstract: First the transmission of information in direct and representative democracies is investigated. Because there is more supply and demand of information, citizens are better informed in direct democracies than in purely representative systems. Then, a survey is given about empirical studies of the economic consequences of direct democracy which show that these consequences are mostly positive. Finally we discuss some of the arguments which are often raised in Germany against the introduction of direct democratic rights on the federal level, especially the reference to 'bad experiences' in the Weimar republic. It is shown that these arguments are not valid. 